\title{
Localized translational motions in semicrystalline Poly(ethylene terephthalate) studied by Incoherent Quasielastic Neutron Scattering
}

\author{
Alejandro Sanz ${ }^{1}$, Tiberio A. Ezquerra ${ }^{1}$, Mari Cruz García-Gutiérrez ${ }^{1}$, Inés Puente- \\ Orench $^{2,3}$, Javier Campo ${ }^{2}$ and Aurora Nogales ${ }^{1}$ \\ ${ }^{1}$ Instituto de Estructura de la Materia, IEM-CSIC, Serrano 121, 28006 Madrid, Spain \\ ${ }^{2}$ Institut Laue-Langevin, BP 156-38042 Grenoble Cedex 9, France \\ ${ }^{3}$ Instituto de Ciencia de Materiales de Aragón, ICMA-CSIC, Pedro Cerbuna 12, 50009 \\ Zaragoza, Spain
}

\begin{abstract}
One of the simplest ways to confine polymeric materials is by self assembling during the crystallization process. The remaining amorphous phase is then constrained by the lamellar crystals. In this manuscript, we aim to shed additional light in the understanding of the amorphous chains dynamics of semicrystalline polymers above the $\mathrm{T}_{\mathrm{g}}$ by using incoherent quasielastic neutron scattering QENS in a mesoscopic timescale $\left(10^{-9}-10^{-10} \mathrm{~s}\right)$ on poly(ethylene terephthalate). The observed dynamics is satisfactorily described by a theoretical model that considers that the proton mobility follows a random jump-diffusion in a restricted environment. We demonstrate that the combination of macroscopic with microscopic dynamic tools allows a complete description of the confined dynamics on a paradigmatic semicrystalline polymer like poly(ethylene terephthalate).
\end{abstract}

\section{INTRODUCTION}

In spite of the numerous studies devoted to the understanding of the mechanism of molecular motions in restricted environments, there are still important questions open to debate, such as the modification of the glass to liquid transition temperature $T_{g}$, distribution of relaxation times and the development of satisfactory theoretical frameworks to describe the confined dynamics among others [1]. Glass forming liquids in general and polymers in particular can be geometrically constrained in different ways, for instance, reducing the sample thickness to the nanoscale, through the location of the material within porous networks and by their mixing with truly nanoparticles [25]. In addition to that, polymeric systems show the ability of self-assembling, one of the main features of soft condensed matter, giving as a result hierarchical morphologies where any of the resulting domains is spatially confined [6]. Phase separated block copolymers and semicrystalline polymers can be considered self-assembled structures 
where the phenomenon of confinement at molecular levels has been widely investigated [7-11]. Semicrystalline polymers consist of lamellar crystals with amorphous domains intercalated in between, in such a way that the disordered chains forming these amorphous domains are formally confined by the laminar crystals [12]. It is well established that polymeric molecules, due to their extremely length and polidispersity, are randomly coiled precluding a complete ordering of the chains, leading to complex and heterogeneous systems. Putting the influence of the lamellar crystals on the amorphous chains dynamics aside, the random coiled scenario of amorphous polymers can be considered a confining environment itself where the polymeric segments are found to be constrained by the surrounding chains and the distance between entanglements may play an important role acting as confining elements. Above the $\mathrm{Tg}$, centre-of-mass diffusion is relatively slow but each chain is able to explore continuously new conformations by internal motions of the main backbone. In this way, atoms in the rubbery state of a polymer undergo a complex type of motion at different time and length scales [13]. The story becomes more complicated when semicrystalline polymers come into play since it has been proposed, on the basis of thermal and dielectric analysis, that a rigid amorphous phase $R A P$, i.e. a phase of amorphous chains lacking segmental motion $[14,15]$, may arise in the interfacial region between the crystalline lamellae and the inter-lamellar amorphous phase. All things considered lead to a highly heterogeneous environment from dynamical and structural points of view.

The main relaxation process observed in glass forming materials is the $\alpha$ mode, only detected above the $T_{g}$. The $\alpha$ relaxation is considered a universal fingerprint of the glass transition phenomenon and it has been principally studied in the macroscopic timescale using relaxation techniques. It is a non-Debye relaxation when observed in the frequency domain (stretched exponential decay in the time domain) and the temperature dependence of its relaxation times strongly departs from the common Arrhenius behaviour. The $\alpha$ relaxation dynamics of semicrystalline polymers, for instance as explored by means of dielectric spectroscopy $D S$, is affected by the presence of crystals, resulting in general in a less intense, broader and slower relaxation [8, 16-20]. In the context of semicrystalline polymers, poly(ethylene terephthalate) PET is one of the most common polymers for industrial necessities and, as a consequence, it has frequently attracted the attention from the scientific community during the last decades. In PET, the crystallization process can be controlled up to some extend playing with 
experimental factors like degree of polymerization, temperature, polydispersity, etc, making possible to study the fully amorphous state or with a controlled amount of crystallinity [7]. All in all makes PET an ideal candidate for the study of the selfconfined dynamics of semicrystalline polymers. We have recently combined dielectric spectroscopy $D S$ with neutron spin echo NSE measurements performed in deuterated and semicrystalline PET, showing that the dynamics of semicrystalline polymers occurs in a homogeneous scenario similar to that valid to describe the dynamics of amorphous polymers [21]. Accordingly, the intermolecular cooperativity is expected to be rather similar in both amorphous and semicrystalline polymers. We proposed that the slowing down of the characteristic segmental relaxation in a semicrystalline polymer in comparison with that of an amorphous one seems to be caused by a retardation of the intramolecular mobility provoked by the anchoring of the polymers chains of the amorphous phase bounded to the crystalline one.

In this manuscript, we look further into the understanding of the amorphous chains dynamics of semicrystalline polymers above the $T_{g}$ by using incoherent quasielastic neutron scattering QENS in a mesoscopic timescale $\left(10^{-9}-10^{-10} \mathrm{~s}\right)$ on fully hydrogenous $P E T$. The observed dynamics is satisfactorily described by a theoretical model that considers that the proton mobility follows a random jump-diffusion in a restricted environment.

\section{EXPERIMENTAL SECTION}

\subsection{Sample details}

Poly(ethylene terephthalate) used here was a commercial sample supplied by RhodiaSter (Rhodia S80, $45.000 \mathrm{~g} / \mathrm{mol}$ ). Films of $275 \mu \mathrm{m}$ were prepared by melt pressing $(540 \mathrm{~K})$ of previously dried pellets and subsequent isothermal annealing at $448 \mathrm{~K}$ was carried out to facilitate the crystallization of the material. The degree of crystallinity was estimated to be around $33 \%$ trough calorimetric measurements. It has been demonstrated that PET shows a heterogeneous crystalline lamellar filling formed by stacks of laminar crystals separated by relatively broad amorphous domains that are commonly called liquid pockets. Inside the stacks of lamellae, we find highly constrained disordered regions with 
average thickness of about $30 \AA$ as estimated by small angle x-ray scattering $S A X S$.

\subsection{Quasielastic neutron scattering}

After making the appropriate corrections to the raw data, the useful quantity in a neutron scattering experiment is given by the double-differential scattering cross section, which gives the probability that a neutron with incident energy $E_{0}$, leaves the scattering material in the solid angle $d \Omega$ about the direction $\Omega$ and with an energy exchange in an interval between $E$ and $E+d E$. Due to the fact that the nucleus-neutron interaction depends on the spin and isotope state of the nucleus, the double-differential scattering cross section contains two parts, namely the incoherent and coherent cross sections respectively.

$$
\frac{\partial^{2} \sigma}{\partial \Omega \partial \omega}=\left(\frac{\partial^{2} \sigma}{\partial \Omega \partial \omega}\right)_{c o h}+\left(\frac{\partial^{2} \sigma}{\partial \Omega \partial \omega}\right)_{i n c} \text { eq } 1
$$

PET contains $C\left(\sigma_{\text {coh }}=5.55\right.$ and $\left.\sigma_{\text {inc }}=0.001\right), O\left(\sigma_{\text {coh }}=4.23\right.$ and $\left.\sigma_{\text {inc }}=0.0008\right)$ and $H$ $\left(\sigma_{\text {coh }}=1.75\right.$ and $\left.\sigma_{\text {inc }}=80.26\right)$. The large value of the scattering incoherent cross section of hydrogen, allowed us to assume that, for PET, the measured quantity is mainly dominated by the incoherent scattering, which is expressed as follows:

$$
\left(\frac{\partial^{2} \sigma}{\partial \Omega \partial \omega}\right)_{i n c}=\frac{k}{k_{0}} \frac{N}{4 \pi} \sigma_{i n c}^{H} S_{i n c}^{H}(Q, \omega) \text { eq } 2
$$

$k$ and $k_{0}$ are the wave-vectors of the incident and scattered neutrons respectively being the modulus of scattering vector $Q=\left|k-k_{0}\right|$ (wave-vector transfer), $N$ is the total number of hydrogen atoms, $\sigma_{i n c}^{H}$ is the incoherent scattering cross section of hydrogen and the function $S_{i n c}^{H}(Q, \omega)$ is the incoherent scattering law associated to the hydrogen atoms. The incoherent scattering function $S_{i n c}^{H}(Q, \omega)$ carries information on the selfcorrelation in space of an individual proton as a function time. $S_{\text {inc }}^{H}(Q, \omega)$ is related through double Fourier transform to the Van Hove self-correlation function [22]. 
Therefore, incoherent neutron scattering on a fully hydrogenous PET sample, provides direct information of the proton dynamics and, consequently, of the whole macromolecule. As a consequence of the limited energy resolution of the neutron spectrometers, what one gets experimentally is the convolution of the incoherent scattering function with the experimental resolution function, that is, the response of the spectrometer when a purely static material is measured and consequently all the neutrons are scattered elastically.

$$
I(Q, \omega)=S_{\text {inc }}^{H}(Q, \omega) \otimes R(Q, \omega) \text { eq } 3
$$

Incoherent quasielastic neutron scattering provides temporal and spatial information of the mechanism of molecular motions, but in order to get a physical insight from the experimental data, a theoretical model must be used to help in the understanding of the microscopic dynamics. The general form of the incoherent scattering law for quasielastic processes is expressed as follows:

$$
S_{\text {inc }}(Q, \omega)=\operatorname{EISF}(Q) \delta(\omega)+S^{q}(Q, \omega) \text { eq } 4
$$

The first term is a purely elastic component and EISF is known as the elastic incoherent structure factor which is always present when the particles move within a restricted environment [22]. The EISF is defined for each value of $Q$ as the ratio of the elastic intensity to the total scattered intensity and provides information on the geometry of the motion [23]. The second term of equation 4 is the quasielastic contribution of the scattering function and contains all the dynamic information. $S^{q}(Q, \omega)$ is generally expressed by a lorentzian function with a half width at half maximum that provides the time scale of the microscopic dynamics and through its $Q$ dependence one can assume a microscopic model of the underlying dynamics. $Q$ is defined as $Q=4 \pi \sin (\theta / 2) / \lambda, \theta$ is the scattering angle and $\lambda$ the neutron wavelength. The lorentzian function is modulated by the term $(1-E I S F)$, in such a way that the model used to fit the experimental data is the following:

$$
S_{\text {inc }}(Q, \omega)=\operatorname{EISF}(Q) \delta(\omega)+\left[(1-\operatorname{EISF}(Q)) \frac{1}{\pi} \frac{\Gamma(\omega)}{\Gamma^{2}(\omega)+\omega^{2}}\right] \text { eq } 5
$$


The convolution of this model function to the experimental resolution data (spectrum at $5 \mathrm{~K}$ ) for each value of $Q$ is what we actually fit to the experimental data in order to extract the half width at half maximum $\Gamma$ (time scale of the motion) of the quasielastic component and the elastic incoherent structure factor EISF which gives information on the geometry of the motion.

QENS experiments were performed at the backscattering spectrometer IN10, at the ILL (Grenoble, France). The incident neutron wavelength was set at $6.28 \AA$ and the $Q$ range was extended from 0.5 to $2 \AA^{-1}$, with an energy resolution at half maximum of $0.45 \mu \mathrm{eV}$ and energy transfer window between -12 and $12 \mu \mathrm{eV}$. Sample thickness was set at 275 $\mu \mathrm{m}$ in order to ensure a transmission of $95 \%$ and minimize as much as possible the poisoning contribution of the multiple scattering effects.

Fixed window scans $(\Delta \mathrm{E} \approx 0)$ were also measured covering a temperature range of $4 \mathrm{~K}$ $<\mathrm{T}<475 \mathrm{~K}$, accessible with a cryofurnace. These measurements monitor the evolution of the elastic scattering intensity as a function of temperature and momentum transfer $Q$, and provide a direct measure of the Debye-Waller factor below the onset of side-group or segmental motions in glassy polymers $[13,22,24]$. The measured elastic scattering intensity $S_{\text {inc }}(Q, \omega \approx 0)$ at constant temperature becomes the $D W$ factor assuming a simple harmonic vibration model:

$$
S_{\text {elastic }}(Q) \approx \exp \left(-Q^{2}\left\langle u^{2}\right\rangle / 3\right) \text { eq } 6
$$

where $\left\langle u^{2}\right\rangle$ is the mean-square amplitude of proton vibrations. In practice, during a window scan experiment, an 'apparent' Debye-Waller factor $\left\langle u^{2}\right\rangle_{a p p}(T)$ is measured as a function of temperature (4-475 $\mathrm{K}$ in the present work). Intensities are normalized to the values corresponding to the lowest measured temperature.

\section{RESULTS AND DISCUSSION}

As it was indicated in the previous section, we first carried out fixed window scans in order to visualize the dynamic behaviour of PET for the IN10 experimental conditions. The panel (a) of figure 1 shows the normalized elastic scattered intensity for 
semicrystalline $P E T$ as a function of temperature for different values of the momentum transfer $Q$. Data are normalized to the elastic intensity at the lowest temperature, in this work $4 \mathrm{~K}$. As a general trend, for the lowest temperature range, the elastic intensity decreases in a linear fashion as the temperature is increased, but for values close to 150 $\mathrm{K}$ a new and deeper dependence is observed and it is probably attributed to the onset of anharmonic vibrations or rotational motions. This second linear trend continues up to approximately $350 \mathrm{~K}$, where the liquid to glass transition of semicrystalline PET is located coinciding with a dramatic drop of the elastic neutron scattered intensity when long range motions of the centres of mass come into play [25]. It is also noticeable that the elastic intensity decreases when the values of $Q$ increase for the whole temperature window. From equation 6 we have estimated the mean square displacement $\left(<\mathrm{u}^{2}>\right)$ of the proton vibrations which are represented in the panel (b) of figure 1. We also include for comparison the values of $\left\langle u^{2}\right\rangle$ for a fully amorphous sample of PET (red triangles). The values of $\left\langle u^{2}\right\rangle$ are rather similar up to the onset of the glass transition for the amorphous and semicrystalline PET, indicating that at least for this energy resolution, the harmonic proton vibrations of PET are not apparently affected by the presence of crystals. For the semicrystalline sample, the glass transition around $350 \mathrm{~K}$ is also manifested by the abrupt increase of the amplitude of the displacements. This change of dependence of $\left\langle u^{2}\right\rangle$ with temperature that characterizes the glass transition takes place at lower temperatures for the amorphous sample $(\sim 340 \mathrm{~K})$. In general, for semicrystalline polymers the glass transition is shifted to higher temperatures as compared to the fully amorphous state as a consequence of the retardation of the segmental motions induced by the presence of the crystals surrounding the amorphous domains $[9,12,26]$. A point worthy of mention is the larger amplitude of the proton displacements for the amorphous $P E T$ above the $T_{g}$, when long range motions become active. That restriction of the dynamics for semicrystalline PET could indicate a confinement effect of the crystalline phase on the amorphous chains dynamics at much localized scales than those characteristic of the segmental motions that typically contribute to the glass transition [12]. Confinement effects on the macromolecular dynamics, also investigated through neutron scattering fixed window scans, have been reported when thin films, polymers intercalated inside porous networks or biological systems are addressed [3, 27-29]. Larger or smaller values of the mean square proton displacement as compared to the bulk are observed for confined systems, especially during the onset of anharmonic motions or long range modes, but also certain variations 
of the pure vibrational dynamics defined by the $D W F$ have been reported on polymer thin films and polymer nano-composites [30-32]. Different physical and geometrical aspects have been considered depending on the case in order to justify these findings. In addition, it is interesting to note that the amorphous PET shows a clear peak in $\left\langle u^{2}\right\rangle$, presumably associated to the cold crystallization process. At higher temperatures, when the initially amorphous PET is already semicrystalline, the amplitude of the localized diffusion-like motions becomes smaller and finally the values of $\left\langle u^{2}\right\rangle$ overlap with those corresponding to the initial semicrystalline sample. Fixed window scans are also convenient to select temperature regions where the dynamics at the corresponding energy resolution is sufficiently active in order to explore the quasielastic spectra. That is the reason we are mainly concerned with the dynamics above the $T_{g}$. QENS spectra were collected at different temperatures and values of momentum transfer $Q$. Figure 2 shows the incoherent scattering functions $S(Q, \omega)$ for semicrystalline $P E T$ as a function of the energy transfer at 350 and $500 \mathrm{~K}$ and at a constant value of $Q=1.85 \AA^{-1}$. QENS data are shown in arbitrary units but without normalization to emphasize the decrease of the central elastic peak at expenses of the QENS broadening when temperature increases. The experimental quasielastic spectra have been described using the equation 5 and the separation between the purely elastic component and the quasielastic broadening is indicated. The spectrum at $350 \mathrm{~K}$ can be simply fit to an elastic component, indicating that at temperatures very close to the glass transition the quasielastic contribution is within the instrumental energy resolution. To illustrate how the dynamic structure factor changes with $Q$, figure 3 presents the experimental data (crosses) of $S(Q, \omega)$ for $500 \mathrm{~K}$ at certain values of the momentum transfer, and the corresponding total fit to equation 5 (red continuous), and the decomposition in terms of the resolution (black dotted) and the lorentzian (blue dashed) functions. The lorentzian function becomes broader when the values of $Q$ increase, showing as a first approach the long range character of the underlying motions [22, 23, 26, 33, 34]. Since the broadening of the dynamic structure factor is directly related to the time scale of the microscopic dynamics, the results shown in figure 3 suggest that the motions causing the quasielastic scattering become faster when the values of $Q$ increase. The best fit of the scattering law defined in terms of the equation 5 to the experimental QENS data allowed us to estimate the $Q$ and temperature dependence of both $\Gamma$ and EISF. The panel $a$ of figure 4 collects the EISF at different temperatures. The EISF is modulated 
by $Q$ in a similar manner for the three temperatures, suggesting that the nature of the dynamics remains unchanged for the whole temperature range. On the contrary, the values of EISF are clearly temperature dependence. The panel $a$ of figure 4 shows that the elastic contribution to the total scattering becomes larger when temperature decreases, finding that is frequently observed for different kind of motions [35-37]. The most accepted explanation for this temperature dependence is connected to the fact the fraction of mobile scatters causing the broadening normally increases with temperature. Before addressing the theoretical description of the EISF by the assumption of the most suitable model, we must pay attention to the $Q$ and temperature dependence of the quasielastic broadening. The main features observed in the panel $b$ of figure 4 are related to the asymptotic values of $\Gamma$ for the high and low $Q$ regimes, specifically for the measurement at $500 \mathrm{~K}$ which shows the largest and best defined broadening. Mobile scatterers in a restricted media are characterized by a non-zero asymptotic behaviour of $\Gamma$ at low $Q$ [22], as it is effectively observed in the lower panel of figure 4. But also, if one look to the high $Q$ limit, $\Gamma$ also tends to a constant value and this is a common feature of motions taking place through jumps between discrete distances instead of by a continuous diffusion [22]. At first look, the dynamics of semicrystalline PET at the mesoscopic time of IN10 seems to be arisen by jumps of mobile protons in a confined media. With these ideas in mind, it is important to remark that each scattering law is conveniently characterized by the most suitable theoretical framework or model that helps to give a physical insight to the experimental QENS data. This work concerns the dynamics of semicrystalline PET above the $T_{g}$. That implies that long-range motions are expected to occur within a restricted media created by the surrounding disordered chains, rigid amorphous domains and laminar crystals. To corroborate our assumptions we have considered the applicability of the long range diffusion in a restricted environment model (DRE model) that was developed by Hall and Ross [22, 38], considering that the motion takes place in one dimension exploring a fixed distance defined by impermeable walls. This model was previously applied by some of us to interpret $Q E N S$ data on a semicrystalline polyketone [35]. The development of this theoretical model proposes the following expression for the EISF:

$$
\operatorname{EISF}_{D R E}(Q, T)=n(T)\left[j_{0}^{2}(Q L(T) / 2)-1\right]+1 \quad \text { eq } 7
$$


where $n(T)$ is the fraction of mobile protons, $j_{0}$ is the spherical Bessel function of first kind and zero order and $L(T)$ is the distance between two rigid and impermeable walls, that is the confinement size. In figure 4(a), the dashed lines represent the theoretical $E I S F$ values predicted by the DRE model, showing an acceptable description of the experimental results. The obtained parameters from fitting the equation 7 to our experimental data are represented in figure 5. Here we see that the confinement size $L$ barely changes with temperature, unlike the fraction of mobile protons $n$ which clearly get larger with temperature, indicating that the higher the temperature, the more mobile protons become active at the mesoscopic timescale $\left(10^{-9}-10^{-10} \mathrm{~s}\right)$. Within the framework of this model, the confinement size is estimated to be around $6.5 \AA$, value situated far below the long spacing (average inter-laminar crystal thickness, $140 \AA$ for semicrystalline PET) for semicrystalline polymers [12, 39]. Therefore, the physical origin of the restricted character of the motions observed in semicrystalline polymers is apparently beyond of the confining effects from the lamellar crystals morphology. The extension of the motions explored here is even smaller than the size of the typical cooperative areas dominated by the segmental mobility, usually $10-20 \AA[6,40]$. Here, for semicrystalline $P E T$, the confinement size remains almost constant for the explored temperature range and this supposes an opposite behaviour than the one observed for poly(ether ether ketone) PEEK where the confining size becomes larger with temperature. PEEK is a much more rigid polymer than PET and consequently more fragile in the context of the classification of glass forming systems proposed by Angell, and therefore is characterized by segmental motions more sensitive to temperature variations $[35,41]$.

As it was already mentioned, the constant values of $\Gamma$ at the high $Q$ values deviates from the Fick's Law and this is a common feature of a jumping mechanism rather than a continuous diffusion. General speaking, jump-diffusion models consider that the diffusing species jump from one site to another after a period of time during which the particle remains fixed apart from vibrations about a centre of equilibrium. After this residence time $\tau$, the particle jumps to a new site, hypothetically in an infinitesimal time [22]. However, the $500 \mathrm{~K}$ data shows a clear deviation from the expected behaviour of the jump-diffusion model calculated by Hall and Ross for the lowest $Q$ value. On the contrary, it is important to note that this deviation is not clearly observed for the lowest temperatures. For these low values of $Q$ and temperature, the quasielastic broadening is not enough to be distinguished from the experimental resolution and, therefore, we are 
not able to estimate confidence values of $\Gamma$. However, there is no reason to postulate a dynamic crossover from 500 to $450 \mathrm{~K}$, so the same departure from the jump-diffusion model at low $Q^{\prime} \mathrm{s}$ is also expected for the 450 and $400 \mathrm{~K}$ data. It has already mentioned that a constant value $\Gamma$ with $Q$, at small $Q$ values, is a common feature of dynamic processes taking place in a spatially confined environment. Therefore, it is not surprising that our EISF data for semicrystalline PET can be reproduced in a satisfactory way under the framework of confined dynamics models.

At this point it is important to note that we have analyzed our EISF data assuming a continuous diffusion within a bounded media between two rigid walls. On the contrary, the evolution of $\Gamma$ with $Q$ seems to indicate that the mobile protons jump from one side to another instead to diffuse in a continuous way. That apparent mismatch can be overcome by the approach developed by Hall and Ross, who calculated the scattering law for jumping motions with a Gaussian distribution of lengths but also including a spatially confined environment $[22,38]$. This model assumes that particles undergo a random-walk between two impermeable walls. When dealing with dynamics within a restricted media, Hall and Ross found the synergy between the one-dimensional diffusion between two walls and the jumping diffusion. A good description of this model and the strategy to calculate the quasielastic broadening at different values of $Q$ is reported elsewhere [35]. The scattering law proposed by Hall and Ross for long range jumping diffusion within a bounded media is described as follows:

$$
S_{\text {inc }}(Q, \omega)=\operatorname{EISF}(Q) \delta(\omega)+\sum_{m=1}^{\infty} A_{m}(Q, L) \ell(\omega, \Gamma(Q, L)) \text { eq } 9
$$

Here the first term is an elastic component with the EISF defined by the equation 7 . The second term is the corresponding quasielastic component which consists of an infinite series of Lorentzian functions $\ell(\omega, \Gamma(Q, L))$, each one with amplitude $\mathrm{A}_{\mathrm{n}}(\mathrm{Q}, \mathrm{L})$ given by:

$$
A_{m}(Q, L)=\frac{(2 Q L)^{2}}{\left[(Q L)^{2}-(m \pi)^{2}\right]^{2}}\left[1-(-1)^{m} \cos (Q L)\right] \text { eq } 10
$$

The half width at half maximum $\Gamma$ of the Lorentzian functions is expressed as follows: 


$$
\Gamma_{m}(Q, L)=\frac{\hbar}{\tau}\left[1-\exp \left(\frac{-m^{2} \pi^{2} r_{0}^{2}}{2 L^{2}}\right)\right] \text { eq } 11
$$

where $\tau$ is the average residence time between two consecutive jumps, $r_{0}$ is the characteristic jump distance and $\hbar=h / 2 \pi$, with $h$ as the Planck constant. Under the assumption of a simple jumping diffusion in an unbounded media, the relation of the residence time $\tau$ with the quasielastic broadening at enough high $Q^{\prime} s$ is simply expressed as $\tau_{Q \rightarrow \infty}=\frac{\hbar}{\Gamma}$. Using this expression we have estimated the residence time $\tau$. The scattering law for jumping diffusion within a restricted volume (equation 9) has been computed using the values of $L$ and $\tau$ and only adjusting the parameter $r_{0}$ (jump distance) in order to fit the experimental data of $\Gamma$ versus $Q$. In practice, for the sum of the lorentzian functions in equation 9, 100 terms were taken into account. Calculations considering more terms do not modify the values of $\Gamma$. The results of these calculations are included in figure 4(b) as continuous lines. The values of $\tau, r_{0}, L$ and the fraction of mobile protons are included in the table 1 . The panel $b$ of figure 4 shows that the segmental motions of semicrystalline PET at the mesoscopic time scale $\left(10^{-9}-10^{-10} \mathrm{~s}\right)$ can be modelled as jumps of distances between 1.8 and $2.5 \AA$ within a restricted environment defined by a characteristic length $L$ of approximately $6.5 \AA$ The residence time at $500 \mathrm{~K}$ has been included in the relaxation map of semicrystalline PET (figure 6) together with neutron spin echo (NSE) and dielectric spectroscopy (DS) data [21]. We have just included the $500 \mathrm{~K}$ data considering that at lower temperatures the $\alpha$ relaxation time scale should fall far away from the IN10 time window as one can observe from the Vogel-Fulcher-Tamman line in figure 6. In this way an extremely broad dynamic range is covered, below and above the $T_{g}$. The dotted line in figure 6 corresponds to the empirical Vogel-Fulcher-Tamman VFT equation that nicely reproduces the cooperative nature of the $\alpha$ relaxation of glass forming systems [41]. We see that the NSE data fall into the VFT line, indicating that the collective dynamics observed through the coherent dynamic structure factor is actually the $\alpha$ relaxation [21]. Interestingly, the data at $500 \mathrm{~K}$ almost coincide for the $N S E$ and backscattering data. In addition to that, the translational character of the dynamics observed in IN10 is also supported by the evolution of $\Gamma$ with the values of $Q$. The reason of the shorter times for 
the IN10 data at 450 and $400 \mathrm{~K}$ than those expected for the $\alpha$ relaxation is presumably attributed to the main contribution to the broadening observed in IN10 of the high frequency tail of the distribution of relaxation times and therefore decoupling from the average $\alpha$ relaxation time, for instance observed by NSE. The better agreement between the NSE data and the VFT line predicted by the low temperature dielectric measurements for the $\alpha$ relaxation confirms that NSE on fully deuterated systems at the maximum $Q$ value of the static structure factor becomes an ideal tool to study selectively the decay of the inter-chain correlations that originate the segmental motions of the $\alpha$ relaxation [42]. On the contrary, IN10 allows exploring the dynamics with excellent momentum transfer dependence from which the geometry of the motions is found, complementing previous NSE and DS results.

\section{CONCLUDING REMARKS}

The dynamics of semicrystalline PET at the microscopic level has been studied by quasielastic neutron scattering $Q E N S$. The quasielastic broadening has been interpreted with a simple lorentzian curve from which the time-scale of the underlying dynamics was estimated. Our EISF data have been modelled assuming that the mobile protons diffuse within a restricted environment. The values of the confinement size were approximately situated between 6 and $7 \AA$ for the diffusion in a restricted environment model. It is interesting to note the agreement between these values with those associated to the typical length scales of the cooperative motions observed above the $T_{g}[43,44]$. We observe an increase of the fraction of mobile protons with temperature. The existence of a rigid amorphous phase at the crystal-amorphous interface that progressively becomes mobile when the system is heated up is the most plausible explanation for the $n(T)$ dependence plotted in figure 6. Similar results have been reported for other semicrystalline polymers $[15,35,45]$. This experimental evidence of the existence of a gradient of mobility caused by the presence of a rigid amorphous phase, which can be consider a new confining factor, supports our data analysis based on the proton diffusion in a restricted environment. The mechanism of the proton dynamics of semicrystalline PET is clearly manifested in the asymptotic behaviour of $\Gamma$ at high values of $Q$, indicating that a jump-diffusion is highly probable to occur. A good description of the experimental data by means of a model that considers that the mobile 
particles undergo random jump trajectories allowed us to estimate the characteristic jump lengths and the residence time between two consecutive jumps. We have successfully applied the approach developed by Hall and Ross in which the scattering particles show a Gaussian distribution of jump lengths within a restricted environment defined by rigid and impermeable boundaries. Here, the values of the jump lengths for $P E T$ are estimated to be slightly lower than those reported for semicrystalline PEEK. To conclude, the inclusion of the IN10 data at $500 \mathrm{~K}$ into the relaxation map of semicrystalline PET indicates that the dynamics observed in this spectrometer is connected to the genuine $\alpha$ relaxation of semicrystalline PET. We demonstrate that the combination of macroscopic with microscopic dynamic tools allows a complete description of the confined dynamics on a paradigmatic semicrystalline polymer like poly(ethylene terephthalate), corroborating previous empirical results by using theoretical models.

\section{Acknowledgments}

This work has been funded by the Spanish MINECO (MAT2009-07789, MAT201123455 and MAT2008-03232). A. S. also acknowledges the Spanish MINECO for financial support and M. Rodríguez for the assistance editing Matlab ${ }^{\circledR}$ codes. The authors thank the Institut Laue-Langevin for beam time and M. A. González for technical assistance during experiments. 
Tables

Table 1. Parameters obtained from the fittings of $\Gamma$ and EISF as a function of $Q$ using the diffusion within a restricted environment model.

\begin{tabular}{ccccc}
\hline \hline $\mathrm{T}(\mathrm{K})$ & $\tau(\mathrm{s})$ & $\mathrm{r}_{0}(\AA)$ & $\mathrm{L}(\AA)$ & $\mathrm{n}$ \\
\hline 400 & $3.5 \times 10^{-10}$ & 1.9 & 6.4 & 0.17 \\
450 & $2.5 \times 10^{-10}$ & 1.8 & 6.1 & 0.28 \\
500 & $1.9 \times 10^{-10}$ & 2.5 & 6.2 & 0.33 \\
\hline \hline
\end{tabular}

Figure Captions

Figure 1. (a) Fixed window scans (at FWHM $0.9 \mu \mathrm{eV}$ resolution) of semicrystalline PET from $\mathrm{T}$ equal to $4-475 \mathrm{~K}$, at selected wavenumbers $\mathrm{Q}\left(\square 0.50 \AA^{-1}\right),\left(\triangle 1.18 \AA^{-1}\right)$, $\left(\diamond 1.68 \AA^{-1}\right)$ and $\left(\triangleright 1.96 \AA^{-1}\right)$. The results are normalized by the elastic intensity measured at $4 \mathrm{~K}$. (b) Apparent mean-square displacement $\left\langle\mathrm{u}^{2}>\left(\AA^{2}\right)\right.$ as a function of temperature for amorphous $(\triangle)$ and semicrystalline $(\bigcirc)$ PET samples. Glass transition temperatures of both species and cold crystallization temperature for initially amorphous PET are indicated by arrows.

Figure 2. Influence of temperature on the experimental QENS spectra on semicrystalline PET at a constant momentum transfer of $1.85 \AA^{-1}$. The total incoherent scattering law (solid red line) is separated into the experimental resolution (dashed black line) and the quasielastic component (dotted blue line).

Figure 3. Q dependence of the incoherent scattering law of semicrystalline PET at 500 K. Solid red lines are the best fit of the equation 5 to the experimental data. Solid black lines correspond to the experimental resolution and the dotted blue lines indicate the lorentzian component, convolved with the resolution function, of the equation 5 that represents the quasielastic broadening. The vertical axes are shown up to a $25 \%$ of the maximum intensity of the central elastic peak.

Figure 4. (a) Experimental data of the elastic incoherent elastic factor EISF as a function of $Q$ for semicrystalline PET. Experimental data of the EISF were estimated from the fits of the QENS spectra to the equation 5. Dashed lines represent the fits of the 
experimental data to the one dimensional diffusion in a restricted environment model (DRE). (b) Half width at half maximum $\Gamma$ of the quasielastic broadening against the momentum transfer $Q$ at different temperatures for semicrystalline PET. Solid lines correspond to the Hall and Ross model (Jump diffusion model in a restricted environment $[35,38]) .400 \mathrm{~K}(\triangle), 450 \mathrm{~K}(\square)$ and $500 \mathrm{~K}(\bigcirc)$

Figure 5. Fraction of mobile protons (left axe) and confinement size (right axe) with temperature for the DRE model which considers rigid walls (equation 7). Lines are guides to the eye.

Figure 6. Relaxation times as a function of reciprocal temperature for semicrystalline PET. The graph collects different relaxation processes like: (a) dielectric $\alpha$ relaxation (O); (b) dielectric $\beta$ relaxation ( $\square$ ); (c) NSE $\alpha$ relaxation ( $\star$ ) and (d) IN10 data (500 $\mathrm{K})$ of the present work $(\triangle)$. Solid red line corresponds to the Arrhenius description of the dielectric $\beta$ relaxation. Dotted black line is the best fit of the dielectric and NSE data of the $\alpha$ relaxation to the Vogel-Fulcher-Tamman equation [17]. Filled pattern corresponds to the IN10 timescale window. 

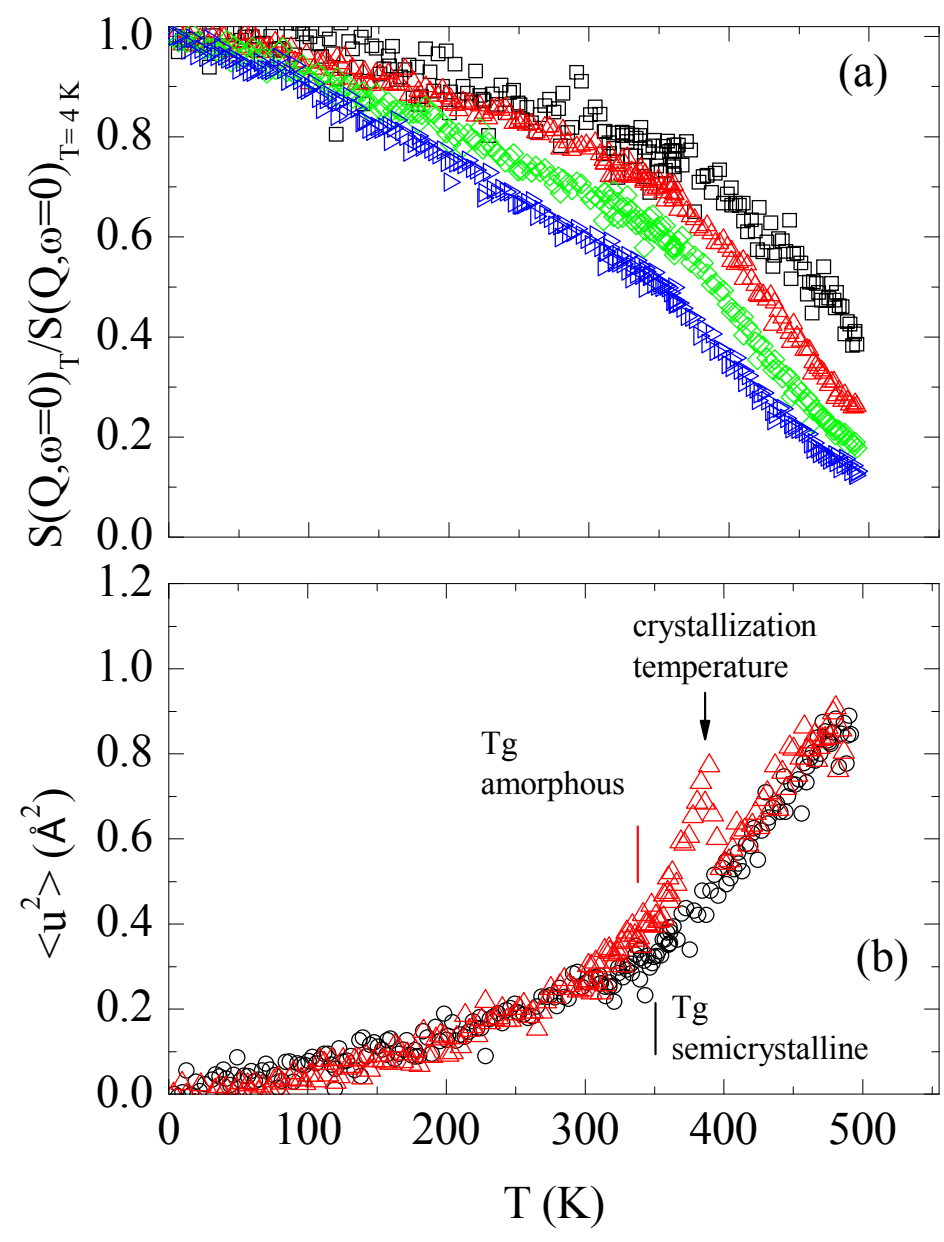

Figure 1 


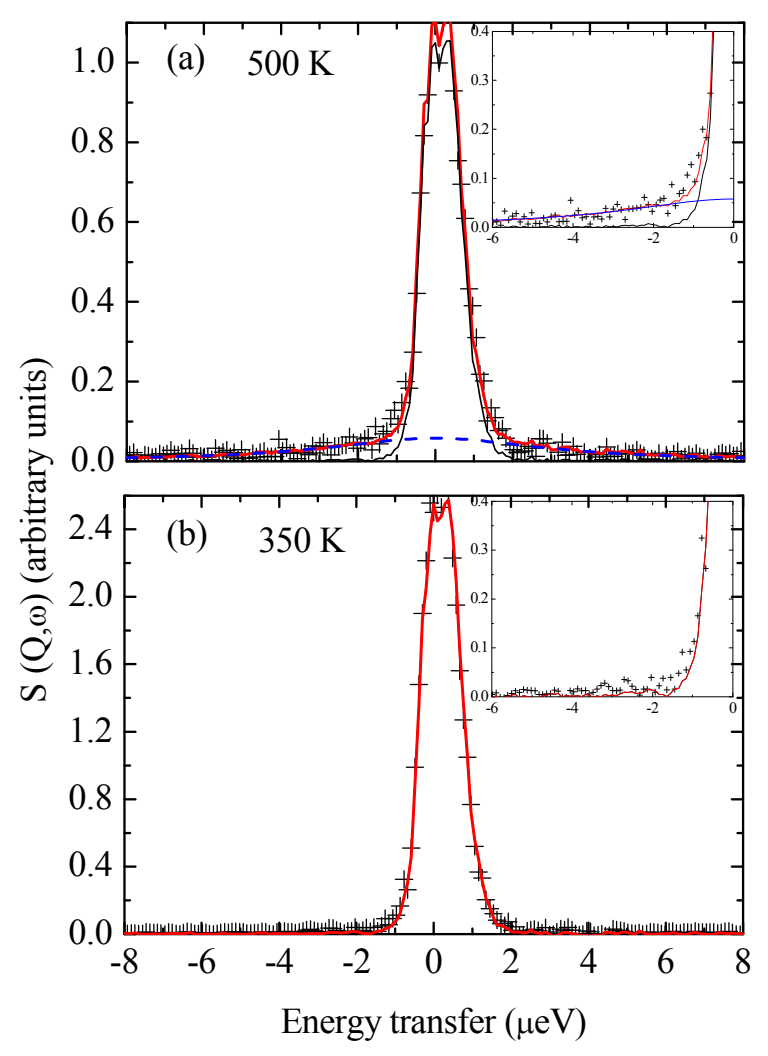

Figure 2 




Figure 3 


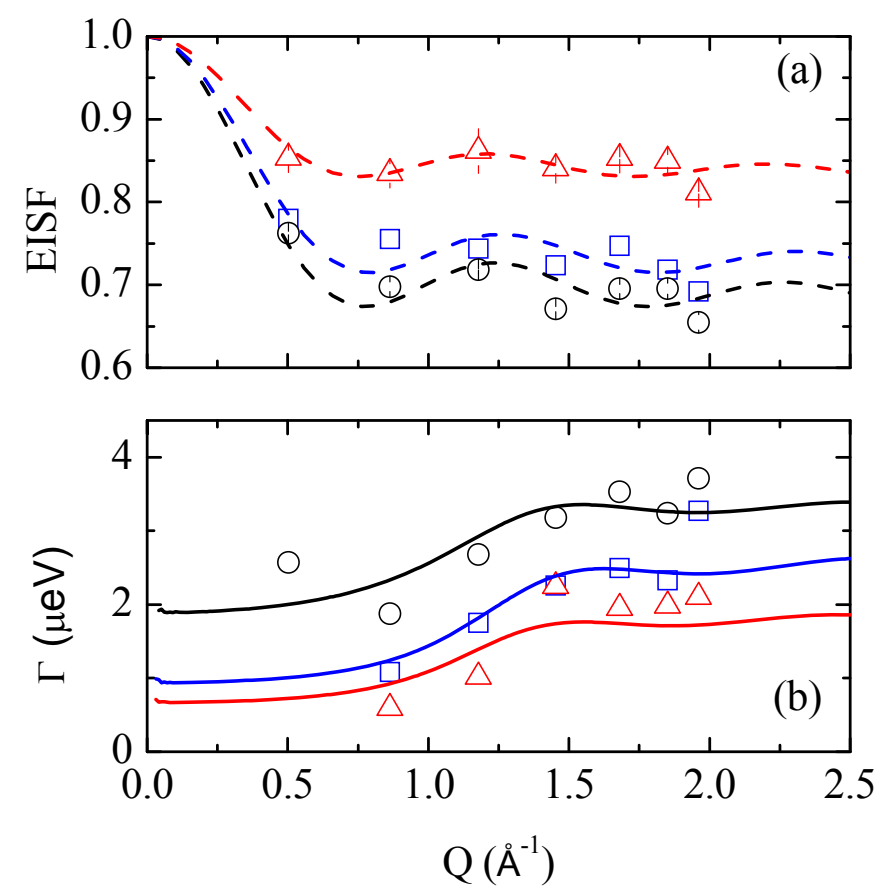

Figure 4 


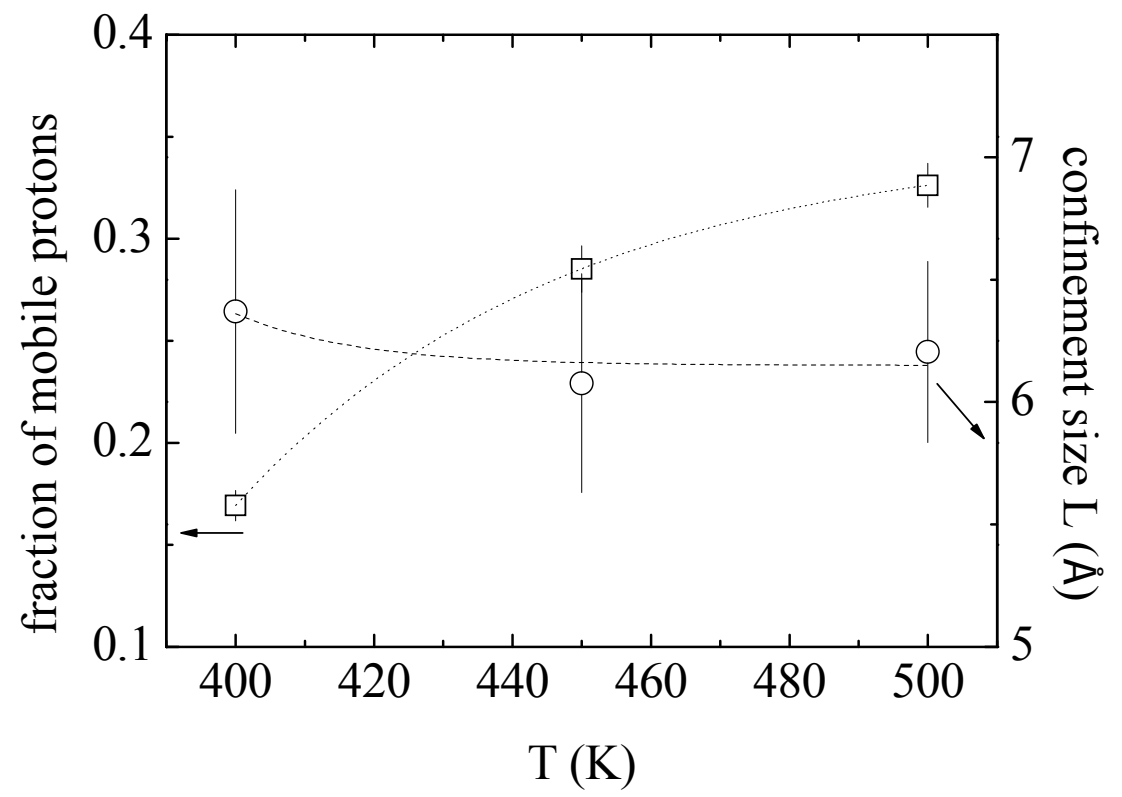

Figure 5 




Figure 6 
1. Alcoutlabi, M. and G.B. McKenna, Effects of confinement on material behaviour at the nanometre size scale. Journal of Physics-Condensed Matter, 2005. 17(15): p. R461R524.

2. Schonhals, A., et al., Glassy dynamics of polymers confined to nanoporous glasses revealed by relaxational and scattering experiments. European Physical Journal $\mathrm{E}$, 2003. 12(1): p. 173-178.

3. Frick, B., et al., Inelastic neutron scattering for investigating the dynamics of confined glass-forming liquids. Journal of Non-Crystalline Solids, 2005. 351(33-36): p. 26572667.

4. Baumchen, O., et al., Reduced Glass Transition Temperatures in Thin Polymer Films: Surface Effect or Artifact? Physical Review Letters. 109(5): p. 5.

5. Wong, H.C., et al., Glass formation and stability of polystyrene-fullerene nanocomposites. Journal of Molecular Liquids. 153(1): p. 79-87.

6. Jones, R.A.L., Soft Condensed Matter. Oxford Master Series in Condensed Matter Physics. 2002, Oxford: Oxford University Press.

7. Coburn, J.C. and R.H. Boyd, Macromolecules, 1986. 19: p. 2238.

8. Soccio, M., et al., Evidence of early stage precursors of polymer crystals by dielectric spectroscopy. Physical Review Letters, 2007. 98(3): p. 037801.

9. Sanz, A., et al., Order and segmental mobility during polymer crystallization: Poly (butylene isophthalate). Polymer, 2006. 47(1281-1290): p. 1281-1290.

10. Lorthioir, C., et al., Heterogeneity of the segmental dynamics of poly(dimethylsiloxane) in a diblock lamellar mesophase: Dielectric relaxation investigations. Macromolecules, 2004. 37(20): p. 7808-7817.

11. Sanz, A., A. Nogales, and T.A. Ezquerra, From hard to soft confinement in a symmetric block copolymer: local and segmental dynamics. Soft Matter. 7(14): p. 6477-6483.

12. Strobl, G., The physics of polymers. Berlin: Springer, 2007.

13. Higgins, J.S. and H.C. Benoît, Polymers and Neutron Scattering. Oxford Series on Neutron Scattering in Condensed Matter. 1996, Oxford: Oxford University Press.

14. Cheng, S.Z.D., M.Y. Cao, and B. Wunderlich, GLASS-TRANSITION AND MELTING BEHAVIOR OF POLY(OXY-1,4-PHENYLENEOXY-1,4-PHENYLENECARBONYL-1,4PHENYLENE). Macromolecules, 1986. 19(7): p. 1868-1876.

15. Huo, P.T. and P. Cebe, TEMPERATURE-DEPENDENT RELAXATION OF THE CRYSTAL AMORPHOUS INTERPHASE IN POLY(ETHER ETHER KETONE). Macromolecules, 1992. 25(2): p. 902-909.

16. Nogales, A., et al., Structure-dynamics relationships of the alpha-relaxation in flexible copolyesters during crystallization as revealed by real-time methods. Journal of Polymer Science Part B-Polymer Physics, 1999. 37(1): p. 37-49.

17. Schönhals. A and Kremer. F, Broad Band Dielectric Spectroscopy, Springer, Berlin, 2002.

18. Sics, I., et al., Cold crystallization of poly(ethylene naphthalene-2,6-dicarboxylate) by simultaneous measurements of $X$-ray scattering and dielectric spectroscopy. Polymer, 2003. 44(4): p. 1045-1049.

19. Sics, l., et al., Simultaneous measurements of small angle $x$-ray scattering, wide angle $x$-ray scattering, and dielectric spectroscopy during crystallization of polymers. Review of Scientific Instruments, 2000. 71(4): p. 1733-1736.

20. Bras, A.R., et al., Influence of crystallinity in molecular motions of poly(L-lactic acid) investigated by dielectric relaxation spectroscopy. Macromolecules, 2008. 41(17): $p$. 6419-6430.

21. Sanz, A., et al., Homogeneous Dynamics within Inhomogeneous Environment in Semicrystalline Polymers. Macromolecules, 2011. 44(20): p. 8124-8128. 
22. Bée, M., Quasielastic neutron scattering: principles and applications in solid state chemistry, biology, and materials science. 1988: Adam Hilger.

23. Bee, M., Localized and long-range diffusion in condensed matter: state of the art of QENS studies and future prospects. Chemical Physics, 2003. 292(2-3): p. 121-141.

24. Frick, B., U. Buchenau, and D. Richter, BOSON PEAK AND FAST RELAXATION PROCESS NEAR THE GLASS-TRANSITION IN POLYSTYRENE. Colloid and Polymer Science, 1995. 273(5): p. 413-420.

25. Frick, B. and D. Richter, THE MICROSCOPIC BASIS OF THE GLASS-TRANSITION IN POLYMERS FROM NEUTRON-SCATTERING STUDIES. Science, 1995. 267(5206): p. 19391945.

26. Hong, P.D., et al., Effect of rigid amorphous phase on glass transition behavior of poly(trimethylene terephthalate). Polymer, 2002. 43(25): p. 6879-6886.

27. Frick, B., M. Koza, and R. Zorn, Dynamics in confinement. European Physical Journal E, 2003. 12(1): p. 3-4.

28. Schonhals, A., et al., Polymers in nanoconfinement: What can be learned from relaxation and scattering experiments? Journal of Non-Crystalline Solids, 2005. 351(3336): p. 2668-2677.

29. Zaccai, G., Biochemistry - How soft is a protein? A protein dynamics force constant measured by neutron scattering. Science, 2000. 288(5471): p. 1604-1607.

30. Inoue, R., et al., Low-energy excitations and the fast process of polystyrene thin supported films studied by inelastic and quasielastic neutron scattering. Physical Review E, 2006. 74(2): p. 8.

31. Sanz, A., et al., Plasticization effect of $C 60$ on the fast dynamics of polystyrene and related polymers: An incoherent neutron scattering study. Journal of Physics Condensed Matter, 2008. 20(10).

32. Soles, C.L., et al., Incoherent neutron scattering as a probe of the dynamics in molecularly thin polymer films. Macromolecules, 2003. 36(2): p. 373-379.

33. Arbe, A. and J. Colmenero, Characterization of the "simple-liquid" state in a polymeric system: Coherent and incoherent scattering functions. Physical Review E, 2009. 80(4): p. 13.

34. Lyonnard, S., et al., Perfluorinated surfactants as model charged systems for understanding the effect of confinement on proton transport and water mobility in fuel cell membranes. A study by QENS. European Physical Journal-Special Topics. 189(1): p. 205-216.

35. Nogales, A., et al., Restricted dynamics in poly(ether ether ketone) as revealed by incoherent quasielastic neutron scattering and broad-band dielectric spectroscopy. Macromolecules, 1999. 32(7): p. 2301-2308.

36. Smuda, C., et al., Methyl group dynamics in glassy, polycrystalline, and liquid coenzyme Q(10) studied by quasielastic neutron scattering. Journal of Chemical Physics, 2008. 129(7): p. 9.

37. Meier, G., F. Fujara, and W. Petry, LOCAL SIDE GROUP-DYNAMICS OF POLY(METHYLPHENYLSILOXANE) (PMPS) AS STUDIED BY QUASI-ELASTIC NEUTRONSCATTERING. Macromolecules, 1989. 22(12): p. 4421-4425.

38. Hall, P.L. and D.K. Ross, INCOHERENT NEUTRON-SCATTERING FUNCTIONS FOR RANDOM JUMP DIFFUSION IN BOUNDED AND INFINITE MEDIA. Molecular Physics, 1981. 42(3): p. 673-682.

39. Nogales, A., et al., Order and Segmental Mobility in Crystallizing Polymers. Lectures Notes Physics. Springer-Verlag Berlin Heidelberg 2007.

40. Hong, L., et al., Molecular cooperativity in the dynamics of glass-forming systems: a new insight. The Journal of chemical physics, 2009. 131(19): p. 194511.

41. Angell, C.A., FORMATION OF GLASSES FROM LIQUIDS AND BIOPOLYMERS. Science, 1995. 267(5206): p. 1924-1935. 
42. Arbe, A., et al., Heterogeneous structure of poly(vinyl chloride) as the origin of anomalous dynamical behavior. Journal of Chemical Physics, 2002. 117(3): p. 13361350.

43. Colmenero, J., et al., TEMPERATURE AND MOMENTUM-TRANSFER DEPENDENCE OF THE DYNAMICS OF THE ALPHA-RELAXATION IN POLYMER MELTS - A QUASI-ELASTIC NEUTRON-SCATTERING STUDY. Physica B-Condensed Matter, 1992. 182(4): p. 369-375.

44. Adolf, D.B. and M.D. Ediger, BROWNIAN DYNAMICS SIMULATIONS OF LOCAL MOTIONS IN POLYISOPRENE. Macromolecules, 1991. 24(21): p. 5834-5842.

45. Sanz, A., et al., Cold Crystallization of Poly(trimethylene terephthalate) As Revealed by Simultaneous WAXS, SAXS, and Dielectric Spectroscopy. Macromolecules, 2010. 43(2): p. 671-679. 